\title{
ANÁLISE DO ÍNDICE DO ARCO PLANTAR EM ESCOLARES DA ZONA RURAL
}

Glauber Carvalho Nobre

Anne Emanuelle da Silva Pereira

Maria Nadja dos Santos Ferreira

Gertrudes Nunes de Melo

Maria do Socorro Cirilo de Sousa

\section{Resumo}

O objetivo deste estudo foi investigar o índice do arco plantar IP e prevalência de desvios posturais no segmento pe em escolares. Decorre um estudo descritivo, transversal em 60 escolares, com média de idade $(11,15 \pm 2,7 \mathrm{dp})$ anos, submetidos ao teste de impressão plantar, equacionando o índice do arco plantar (IP), que estabelece a relação entre a região central e posterior dessa impressão, referenciando em Cavanagh e Rodgers (1987). Utilizou-se do programa SPSS versão 13.00 para estatística descritiva e inferencial. Não foram observadas diferenças significativas do IP dos lados direito e esquerdo quando comparado por dominância do segmento, entre e intergênero. A maioria dos escolares, tanto do sexo masculino quanto do feminino, indicou estar em índices classificatórios considerados normais.

\section{Palavras-Chave}

Escolares da zona rural; Pés, Impressão plantar.

\section{ANALYSIS OF CONTENTS OF PLANTAR ARCH IN SCHOOL OF RURAL AREA}

Glauber Carvalho Nobre

Anne Emanuelle da Silva Pereira

Maria Nadja dos Santos Ferreira

Gertrudes Nunes de Melo

Maria do Socorro Cirilo de Sousa

\begin{abstract}
This study aimed to examine the plantar arch of the IP index and prevalence of postural deviations in segment eg in school. It follows a descriptive study, transverse in 60 schools, with average age $(11.15 \pm$ 2.7 SD) years, subject to the test print plant, considered the index of the plantar arch (IP) which establishes the relationship between the central region and further that impression, referencing in Cavanagh and Rodgers (1987). We used the program SPSS version 13:00 to descriptive and inferential statistics. There were no significant differences of the IP right and left when compared to the dominant segment, and between intergeneric. The majority of students, both male as female, said to be in classificatory indices considered normal.
\end{abstract}

\section{Key-Words}

Schoolchildren; Feet; Printing plant. 


\section{INTRODUÇ̃̃̃O}

Atualmente, está cada vez mais difícil se manter em uma postura adequada, devido às grandes sobrecargas que o corpo vem sofrendo no dia-a-dia, tais como, o uso de mochilas, calçados, a carga horária excessiva de trabalho, entre outros, o que podem desencadear com o tempo, um desequilíbrio em algumas estruturas corporais, podendo acarretar problemas de saúde. Percebe-se ainda que uma parte corporal que sofre muito com todas essas alterações posturais são os pés, que por sua vez tem como função possibilitar harmonia corporal e o deslocamento.

Sabe-se que durante toda a evolução humana, o homem desenvolveu uma característica na sua maneira de se deslocar e de se manter estático no meio, sendo essa função uma das atividades mais simples e necessária à vida diária (GALLAHUE; OZMUN, 2005; PAYNE; ISAACS, 2001). Esta por sua vez, é desenvolvida logo nos primeiros anos de vida e conforme Delisa (1992), o padrão de marcha bípede do ser humano é adquirida na infância por volta dos 07 ou 08 anos, onde o sistema sensório-motor está se adaptando a gerar automaticamente um conjunto repetitivo de comandos de controle motor que permiti uma pessoa caminhar sem esforço consciente.

Assim dentre as habilidades fundamentais utilizadas pelo homem, Amadio (1996) relata que o andar é uma das mais importantes, pois possibilita através da locomoção, a exploração dos espaços em diferentes contextos. Este envolve distintos padrões de movimentos estabelecidos por complexas estruturas neurológicas sincronizadas com as demais funções do aparelho locomotor, possibilitando de certa forma a construção de outros vários movimentos e habilidades mais especificas.

Percebendo deste modo que os movimentos da locomoção se apresentam de forma variáveis, visto que, cada indivíduo apresenta particularidades sobrepostas ao padrão básico de locomoção e que se define com o tempo de vida. Lippert (1996) ressalta que cada indivíduo tem um padrão de marcha que representa uma maneira de deslocar-se no ambiente, de forma aceitável, com menor esforço físico e uma estabilização adequada.

Assim, os pés formam bases de apoios para locomoção e apresentam uma relação profunda com a postura, e ainda se responsabiliza pela estática e dinâmica do corpo, suportando o peso corporal, como 
também colaborando com o amortecimento durante os gestos de marcha e a corrida. Deste modo com a presença de desarmonia neste segmento corporal pode vim a acarretar dor, desconforto e até incapacidades.

Em se tratando dos efeitos clínicos, Smith et al. apud Otowicz (2004) divide o pé também em três partes: Retropé: formado pelo tálus e calcâneo, Médiopé: formado pelo navicular, cubóide e os três cuneiformes, Antepé: formado pela parte medial e distal dos metatarsianos e pelas falanges. Desta forma a medida da região do médio pé serve como parâmetro para definir o tipo de pé.

Portanto, quando há alguma deformação nestes tais como, o pé valgo - onde ocorre uma pronaçãoeversão, na qual o peso do corpo age deprimindo o arco plantar, ou ainda o pé varo - onde ocorre uma supinação-inversão de modo que o peso é transferido para a face externa do pé e seu lado medial fica fora do solo, entre outras deformidades que muitas vezes se não diagnosticada e tratada, com o passar dos anos pode causar sérios problemas de saúde.

De um modo geral ao passo que uma criança cresce e se desenvolve, as modificações somáticas quantitativas em conjunto com os processos de diferenciação estrutural, de acordo com suas necessidades diárias, seus calçados e seus hábitos de vida, podem formar uma resposta típica e diferenciada da marcha, constituindo o seu padrão motor (MALINA, 2004; PRETA; GOMES, 2004). Assim dependo desta resposta típica, podem surgir posturas equivocadas durante as atividades ou tarefas que são realizadas freqüentemente, podendo levar a uma aceleração do processo de desgaste em estruturas especifica do aparelho locomotor (STABELINI NETO et al., 2004)

Percebe-se ainda que é durante a transição da infância até o fim da adolescência onde ocorrem as maiores modificações no processo de equilíbrio que é essencial para o ato de se descolar e de se manter em posição ortostática. Desta forma, o pé muda não somente suas dimensões físicas, mas também seu aspecto e forma, podendo haver grandes variações, devidos os hábitos de vida adotados, entre faixas etárias e/ou dentro de uma mesma faixa etária, entre gêneros e assim por diante.

Para tanto existe algumas maneira de acompanhar e analisar o desenvolvimento plantar, segundo Manfio et al. (2007), dentre os diversos métodos de analise plantar, um dos mais comuns é o índice do arco plantar instituído por Staheli, que utiliza impressões plantares expostas em folhas de papel. Essa é uma Conexões: revista da Faculdade de Educação Física da UNICAMP, Campinas, v. 7, n. 2, p.1-12, maio/ago. 2009. 
técnica indireta, relativamente simples e facilmente realizável, utilizada como um método de avaliação quantitativa devido às suas vantagens e efetividade na aplicação clínica, como baixo custo no sistema de obtenção de imagens.

Contudo de posse dessas informações tornou-se viável a analise das características do segmento pé, se utilizando do método acima citado e reconhecendo que são poucos os estudos com escolares nesta fase da vida. Considerando o pé como fundamental para locomoção humana, assim como para a execução de tarefas independentes, o estudo de suas características é tema de grande interesse para a área da saúde, justificando a realização desta pesquisa, a fim de colaborar com os profissionais diretamente ligados.

Acredita-se ainda que a partir das análises posturais com os escolares, os professores de Educação Física e os demais profissionais envolvidos com a educação terão condições de orientar os alunos para a adoção de posturas corretas nas atividades do dia-a-dia, como medida de prevenção de possíveis desvios e, caso necessário, encaminhá-los a um profissional especialista.

Desta forma a questão norteadora deste estudo é: Quais são os índices de arco plantar e prevalência de desvios posturais do segmento corporal pé de escolares da zona rural? Será que existe diferença do índice do arco plantar entre gênero? Neste sentido o objetivo deste estudo é investigar o índice do arco plantar em escolares da zona rural da cidade de Juazeiro do Norte - CE, buscando identificar os tipos de pés mais presentes entre os gêneros.

\section{MATERIAL E MÉTODOS}

Caracterização da pesquisa: Trata-se de um estudo do tipo transversal, quantitativos, de caráter descritivo e de campo. O estudo foi desenvolvido em uma escola de ensino fundamental I e II, pertencente à rede pública de ensino, localizada na zona rural da cidade de Juazeiro do Norte - CE.

População e amostra: A população da pesquisa foi composta de indivíduos na fase escolar. A amostra caracterizou-se como sendo probabilística, aleatória simples, sendo constituída de 60 estudantes de um universo de 153 escolares, que representa $39,21 \%$ do total de alunos, sendo 37 do sexo masculino $(61,7 \%)$ e 23 do feminino (38,3\%), regularmente matriculados na Escola Municipal de Ensino Fundamental I e II 03 de Junho, com idade de 5 a 17 anos, de ambos os gêneros. A opção pela faixa etária citada anteriormente foi intencional, uma vez que as mudanças do segmento pé acontecem mais Conexões: revista da Faculdade de Educação Física da UNICAMP, Campinas, v. 7, n. 2, p.1-12, maio/ago. 2009. 
precisamente na transição da infância a adolescência.

Instrumentos: $O$ material utilizado foi constituído de, uma ficha para coleta de dados contendo informações de identificação pessoal como nome, idade, sexo, série entre outros, utilizou-se ainda folha de papel oficio A4, tinta guache preta de fácil remoção, rolo pequeno para pintar os pés, a fim de adquirir a impressão plantar, papel toalha e material de expediente (pranchetas, lápis, borrachas, bacias, grampeador e grampo, envelope A4).

Procedimentos para a coleta de dados: Após a elaboração, o projeto foi submetido a um estudo piloto com a participação de crianças de 5 a 7 anos de idade, a fim de verificar a sua aplicabilidade. Em seguida entrou em contato com a direção da escola como também com o profissional de educação física atuante na escola. Após a autorização foi feito um contato com os profissionais que contribuíram com pesquisa durante a coleta dos dados agendando um treinamento onde foram apresentados os objetivos do estudo como também todo o procedimento a ser seguido.

Entrou-se em contato com os pais ou responsáveis pelos alunos através de uma reunião na escola marcada pelo professor de educação física e direção da escola, assim foi apresentado o objetivo da pesquisa e como procederia a coleta de dados. Em seguida foi agendado o horário e local para as coletas e entrega do Termo de Consentimento Livre e Esclarecido (TCLE) que deveria ser assinado e devolvido no dia da coleta por cada participante/aluno.

No dia e horário marcado, com de posse do TCLE assinado pelos responsáveis, foi preenchida a ficha de dados com a identificação pessoal de cada participante, descrevendo nome, idade, sexo, série, turma, endereço e contatos e em seguida para obtenção das impressões plantares utilizou-se o teste do plantigrama, onde o estudante enquanto sentando, tinha seus pés pintado e todo o procedimento esclarecido passo a passo pelo avaliador.

Assim com o auxilio do examinador, solicitava-se ao avaliado que levanta-se lentamente de modo a apoiar ambos os pés nas folhas de papel logo abaixo destes, posteriormente era solicitado que a criança e/ou jovem volta-se a posição sentado de modo a retirar um pé de cada vez da folha com ajuda do examinador a fim de evitar deslize e conseqüentemente a invalidez do exame.

Em seguida avaliou o índice do arco plantar, que por sua vez estabelece uma relação entre a região central Conexões: revista da Faculdade de Educação Física da UNICAMP, Campinas, v. 7, n. 2, p.1-12, maio/ago. 2009. 
$\overline{\text { e posterior do pé e é calculando como se segue. Traça-se uma linha tangente na borda medial do ante-pé e }}$ na região do calcanhar. Calcula-se o ponto médio dessa linha e partindo desse ponto em seguida traça-se uma linha perpendicular que cruza a impressão plantar e outra na tangência do calcanhar. Assim se obtém a medida da largura do apoio da região central do pé (B) e da região posterior ou calcânea do pé (C) em milímetros. Assim o Índice do Arco Plantar é calculado dividindo o valor da região central "B" pela região do calcanhar "C" ou seja, (IAP = B/C), segundo Henandez et al. (2006).

Para classificação do pé foi utilizado o método descrito por Cavanagh e Rodgers apud Teodoro et al. (2007). Um eixo longitudinal foi desenhado do centro do calcanhar até o centro do segundo dedo, correspondendo respectivamente às linhas " $\mathrm{k}$ " e "j", em seguida duas linhas perpendiculares foram desenhadas, uma na base do calcanhar e a outra na base da região anterior do pé, excluindo os dedos, a distância entre essas linhas é representada por "L", que foi dividida em três partes iguais, representadas por "A", "B" e "C".

Através da obtenção do coeficiente de razão das áreas do pé, foi determinada a classificação do arco plantar. O parâmetro utilizado para classificar o tipo do pé foi como se segue, $\mathrm{A}$, representa a área da região anterior do pé (antepé), B, a área da região medial do pé (mesopé) e C, a área da região posterior do pé (retropé). $\mathrm{O}$ calculo é feito dividindo a região medial do pé (B) pela soma das três regiões $(\mathrm{A}+\mathrm{B}+$ C). Sendo assim, o pé classificado como cavo aquele com o coeficiente menor que 0,07 , em normal entre 0,08 e 0,24 e em plano entre 0,25 e 0,36 .

Plano analítico: Após os registros dos dados manualmente foi confeccionado um banco de dados em pacote estatístico Statiscal Package For Science Social (SPSS) versão 13.00 e aplicado à estatística Descritiva de medidas de tendência central e dispersão com média, desvio padrão, valores máximos e mínimos. Para estatística Inferencial, utilizou-se do testes " $t$ " de Student para amostras independentes. O nível de significância adotado foi de 5\%. Apresentou-se os dados em tabelas e gráficos.

\section{APRESENTAÇÃO DOS RESULTADOS E DISCUSSÃO}

Percebe-se que o pé humano é uma estrutura altamente especializada e de grande importância para o suporte, locomoção, sustentação e equilíbrio do corpo tanto em posição estática como em marcha, sabe-se também que esta estrutura pode apresentar classificações distintas como pé: plano ou chato, cavo, valgo, calcâneo, eqüino, adutor e abdutor. 
Para tanto, buscou inicialmente analisar o resultado pela estatística descritiva utilizando a média, desvio padrão, valores mínimos e máximos das variáveis ante-pé direito e esquerdo (AD/AE) médio pé direito e esquerdo (MD/ME) retro-pé direito e esquerdo $(\mathrm{RD} / \mathrm{RE})$ e ainda o coeficiente do arco plantar de ambos os pés (CAP-D/CAP-E), ou também chamado de índice de arco plantar, do grupo $\mathrm{N}=60$. Após as analise foi observado que não houve diferenças significativas do coeficiente plantar, onde para o lado direito e esquerdo a média foi de 0,18 (TABELA 1).

TABELA 1 - Valores de média, desvio padrão, mínimo e máximo das medidas da região do ante-pé direito (AD) e esquerdo (AE), médio pé direito (MD) e esquerdo (ME), retro-pé direito (RD) e esquerdo (RE), coeficiente do arco plantar direito (CAP-D) e esquerdo (CAP-E) $(\mathrm{N}=60)$.

\begin{tabular}{ccccccccc}
\hline & AD & MD & RD & CAP-D & AE & ME & RE & CAPE \\
\hline $\mathbf{X}$ & 8,00 & 2,79 & 4,55 & 0,18 & 8,18 & 2,83 & 4,65 & 0,18 \\
DP & 0,92 & 0,96 & 0,70 & 0,06 & 0,98 & 1,01 & 0,70 & 0,06 \\
Mín & 6,30 & 0,00 & 2,50 & 0,00 & 6,10 & 0,00 & 3,30 & 0,00 \\
Max & 10,10 & 4,80 & 6,20 & 0,32 & 11,10 & 5,30 & 6,50 & 0,35 \\
\hline
\end{tabular}

X- Média; DP- Desvio padrão; Min. - Valor mínimo; Max. - Valor máximo

Já no estudo de Hernandez et al. (2006), onde buscou verificar e avaliar o índice do arco plantar e a ocorrência de pés planos em crianças de 5 a 9 anos de idade, percebeu que houve diferenças significativas entre os lados, sendo que os índices plantares médios foram de 0,67 para o lado direito e de 0,61 para o esquerdo.

$\mathrm{Na}$ analise de Filippin et al. (2007) objetivando determinar se há diferenças na distribuição de pressão lantar estática e dinâmica entre crianças obesas e eutróficas, com idades entre nove e onze anos. Constatou-se que as crianças obesas apresentaram maiores áreas de contato, picos de pressão, pressões médias máximas e integrais pressão-tempo, quando comparadas às eutróficas, com diferenças significativas, principalmente nas regiões do médio-pé e antepé.

Num segundo momento notou as diferenças do índice plantar por gênero e percebeu que quando comparados separadamente também não houve diferenças, mesmo as meninas apresentando um valor maior do índice do arco plantar que os meninos. (TABELA 2). 
TABELA 2 - Valores de média, desvio padrão, mínimo e máximo do coeficiente do arco plantar direito (IAC-D) e esquerdo (IAC-E) de acordo com gênero ( $N=60)$.

\begin{tabular}{lcccc}
\hline & \multicolumn{2}{c}{ Masculino } & \multicolumn{2}{c}{ Feminino } \\
\cline { 2 - 5 } & CAP-D & CAP-E & CAP-D & CAP-E \\
\hline Média & 0,18 & 0,18 & 0,17 & 0,17 \\
Desvio padrão & 0,05 & 0,05 & 0,05 & 0,05 \\
Mínimo & 0,0 & 0,0 & 0,0 & 0,0 \\
Máximo & 0,32 & 0,35 & 0,25 & 0,26 \\
p & & 0,458 & & \multicolumn{2}{c}{0,578} \\
\hline
\end{tabular}

Na pesquisa de Volpon (1993) com o objetivo de entender melhor a evolução etária do arco plantar, analisando o crescimento do pé segundo as impressões plantares, incluídos 637 sujeitos hígidos, de ambos os sexos, na faixa etária de 0-15 anos, Observou que as curvas para o sexo masculino e feminino são semelhantes até os 12 anos de idade. A partir daí, o comprimento da impressão plantar estabiliza-se na mulher e, no homem, sofre um pico dos 13 aos 14 anos. Ambas as curvas têm maior inclinação até os dois anos de idade.

Este ainda identificou que até os dois anos de idade, a média é alta $(0,80)$ significando arco plantar virtualmente ausente. Depois dessa idade, o índice de contato (IC) diminui rapidamente até os seis anos de idade, para praticamente estabilizar-se até os 15 anos. E que o índice maior de pés planos ocorre até os dois anos de idade. Após os seis anos, situa-se em torno de dois por cento. Em contrapartida, a incidência de pé cavo é maior aos dez anos de idade.

Assim quando analisado e classificados os pés do grupo nota-se pelo Gráfico 01, que boa parcela dos avaliados, tanto para o pé direito quanto para o esquerdo, estão enquadrados dentro da normalidade, segundo a classificação adotada por Cavanagh e Rodgers (1987) citado por Teodoro et al. (2007).

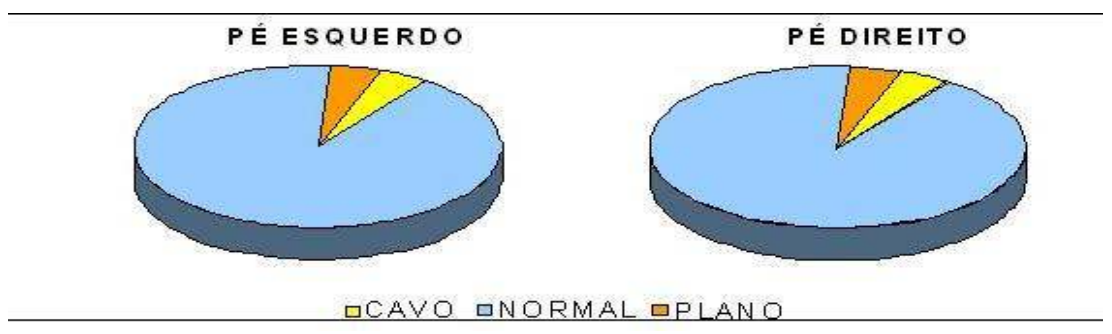

GRÁFICO 1: Classificação percentual dos desvios posturais do segmento pé $(\mathrm{N}=60)$.

Conexões: revista da Faculdade de Educação Física da UNICAMP, Campinas, v. 7, n. 2, p.1-12, maio/ago. 2009. 
Observando a mesma analise com os valores em percentuais e dividindo por gênero e representado em tabela, percebe-se que as meninas apresentam valores semelhantes aos dos meninos, mas notando também que os pés esquerdos e direitos dos meninos encontram-se classificado como plano em maior escala. Viladot (1987) citado por Otowicz (2004), descreve como sendo o tipo cavo o pé que apresenta uma medida menor que um terço da medida do antepé e do tipo plano o pé que apresenta uma medida maior que um terço da medida do antepé.

\section{TABELA 3 - Percentual de classificação dos pés de acordo com gênero ( $N=60)$}

\begin{tabular}{lcccc}
\hline & \multicolumn{2}{c}{ Pé Esquerdo } & \multicolumn{2}{c}{ Pé Direito } \\
\cline { 2 - 5 } Cavo & Feminino & Masculino & Feminino & Masculino \\
Normal & $4,35 \%$ & $2,7 \%$ & $4,3 \%$ & $2,7 \%$ \\
Plano & $91,3 \%$ & $86,5 \%$ & $91,3 \%$ & $89,2 \%$ \\
\hline
\end{tabular}

No estudo de Ferreira (2007), quando buscou analisar a prevalência de desvios posturais em escolares da zona urbana e rural na cidade de Crato, Ceará, através de fotografias, identificou numa visão posterior que o segmento pé e tornozelo, apresentaram maiores tendências a pés varos e valgos para ambos os grupos. Já na visão lateral e visão anterior encontrou-se normalidade para ambas as partes.

Já no estudo de Silva et al. (2005), objetivando analisar a prevalência de alterações posturais na avaliação diagnóstica para prescrição e implementação de um programa de exercícios, em 200 pessoas com uma média de idade 29,24+12,70 anos, de ambos os gêneros, encontrou-se que na prevalência de alterações do segmento corporal pés há uma diferença quando analisado entre os gêneros $(p=0,000)$ e entre as faixas etárias $(p=0,000)$, e também em cada gênero e suas respectivas faixas etárias $(p=0,000)$.

E ainda neste mesmo estudo, expressando os valores em percentual observaram que $36 \%$ das mulheres e $15 \%$ dos homens estão com o segmento pés simétricos, outros 7\% das mulheres e $10 \%$ dos homens estão classificados com pés valgos, notando que os homens vêm apresentando mais alterações nesse segmento que as mulheres. 
Sabe-se ainda que estas características podem sofre influencia pelos hábitos diários e estilo de vida, como por exemplo, pelo tipo de calçado usado, a forma de transportar implementos como mochilas, a própria composição corporal, entre outros, o que não foi elementos de observação neste estudo.

\section{CONCLUSÕES}

$\mathrm{O}$ estudo com base na amostragem permitiu concluir que não foram observadas diferenças significativas do coeficiente plantar para o lado direito e esquerdo quando comparados o grupo e por gêneros e quanto à classificação dos pés ambos os gêneros estão em maior escala normais. A avaliação diagnóstica nas escolas para o acompanhamento e o desenvolvimento de exercício físico realizado principalmente pelos professores de Educação Física é capaz de encontrar diferentes alterações corporais dando-lhes suporte e segurança durante suas intervenções.

\section{REFERÊNCIAS}

AMADIO, A. C. Fundamentos biomecânicos para análise do movimento humano. São Paulo: Laboratório de Biomecânica: EEFUSP, 1996.

BARELA, J. A. Estratégias de controle em movimentos complexos: ciclo percepção-ação no controle postural. Rev Paul Ed Fis, São Paulo, supl. 3, p.79-88, 2000.

BRUNO, M. Avaliação médica e física para atletas e praticantes de atividades físicas. São Paulo: Roca, 2000.

CARNAVAL, P. E. Medidas e avaliação em ciência do esporte. 6. ed. Rio de Janeiro: Sprint, 2004.

FERREIRA, M. N. D. S. Prevalência de desvios posturais em escolares das zonas urbana e rural na cidade de Crato, Ceará. 2007. Monografia (Trabalho de Conclusão de Curso Superior de Tecnologia em Gestão Esportiva) - Centro Federal de Educação Tecnológica Unidade de Ensino Descentralizada de Juazeiro do Norte, Juazeiro, 2007.

GALLAHUE, D. L.; OZMUN, J. C. Compreendo o desenvolvimento motor: bebês, crianças adolescentes e adultos. 3. ed. São Paulo: Phorte, 2005

LIPOSCK, D. B.; ROSA NETO, F.; SAVALL, A. C. Validação do conteúdo do instrumento de avaliação postural - IAP. Disponível em: http://www.efdeportes.com/Revista Digital, Buenos Aires, ano 12, n. 109, jun. 2007.

MALINA, R. M. Motor Development during infancy and early childhood: Overview and suggested directions for research. Int J Sport and Health Sci, v. 2, p. 50-66, 2004. 
PAYNE, V. G.; ISAACS, L. D. Human motor development: a life approach. California: Moutan View, 2001.

PRETA, C.; GOMES, J. C. Desenvolvimento de padrões motores: a marcha. 2004. Dissertação (Mestrado) - Faculdade de Motricidade Humana, Universidade Técnica de Lisboa, Lisboa, 2004.

STABELINI NETO, A. et al. Relação entre fatores ambientais e habilidades motoras básicas em crianças de 6 e 7 anos. Rev Mackenzie de Ed Fis Esp, v. 3 n. 3, p. 135-140, 2004.

TEODORO, E. C. M.; TOMAZINI, J. E.; NASCIMENTO, L. F. C. Hálux valgo e pés planos: as forças plantares são iguais? Acta Ortop Bras, São Paulo, v. 15, n. 5, 2007. 


\title{
Glauber Carvalho Nobre \\ Universidade do Estado de Santa Catarina
}

\begin{abstract}
Anne Emanuelle da Silva Pereira
Universidade Castelo Branco, Instituto de Educação Ciências e Tecnologia do Ceará
\end{abstract}

Maria Nadja dos Santos Ferreira

Universidade Castelo Branco, Instituto de Educação Ciências e Tecnologia do Ceará

\author{
Gertrudes Nunes de Melo \\ Instituto de Educação, Ciências e Tecnologia do Ceará
}

Maria do Socorro Cirilo de Sousa

Universidade Federal da Paraíba/Universidade Castelo Branco

\section{Referência do artigo:}

\section{ABNT}

NOBRE, G. C. et al. Análise do índice do arco plantar em escolares da zona rural. Conexões, v. 7, n. 2, p. 1-12, 2009.

\section{APA}

Nobre, G. C., Pereira, A. E. S., Ferreira, M. N. S., Melo, G. N., \& Sousa, M. S. C. (2009). Análise do índice do arco plantar em escolares da zona rural. Conexões, 7(2), 1-12.

\section{VANCOUVER}

Nobre GC, Pereira AES, Ferreira MNS, Melo GN, Sousa, MSC. Análise do índice do arco plantar em escolares da zona rural. Conexões, 2009; 7(2): 1-12.

Recebido em: 25/04/2009

Aceito para publicação em: jun. 2009 